\title{
MATEMÁTICA FRACTAL
}

\section{FRACTAL MATHEMATICS}

\author{
AUTORES \\ Daviz Melero: Alumno de la Escuela Politécnica. Universidad de Alcalá \\ david.melero@aol.com
}

\section{CURRÍCULUM VITAE}

Profesor de IE University, miembro también de la Comisión permanente de la CITT (Comisión de Ingenieros Técnicos de Telecomunicaciones).

\section{RESUMEN}

La geometría fractal provee una descripción y una forma de modelo matemático para las aparentemente complicadas formas de la naturaleza. Éstas poseen a veces una remarcable invariancia a la simplificación bajo los cambios de la magnificación, propiedad que caracteriza a los fractales como veremos más adelante.

\section{PALABRAS CLAVE}

Matemática - Fractal - Geometría

\begin{abstract}
Fractal geometry provides a description and a mathematical model for the seemingly complicated forms of nature. They sometimes have a remarkable simplification
\end{abstract}


invariance under changes of magnification, a property that characterizes fractals as we shall see.

\section{KEY WORDS}

Mathematics - Fractal - Geometry

Para este número doble, especial de la Navidad, hemos hecho una excepción introduciendo un artículo de divulgación un poco largo, lo que hará que el tiempo de carga sea grande. Pero el gran interés del mismo, escrito en un lenguaje asequible al totalmente profano, hace que merezca la pena. Lector no te asustes de la extensión, aquí se encierra uno de los más bellos aspectos de la matemática moderna

"La Geometría Fractal no es solamente un capítulo de las matemáticas, sino aquél que ayuda a todo el mundo a ver el mismo viejo mundo de una manera distinta".

Benoit Mandelbrot

La geometría tradicional, la euclídea, es la rama de la matemática que se encarga de las propiedades y de las mediciones, tales como puntos, líneas, planos y volúmenes. La geometría euclídea también describe los conjuntos formados por la unión de los elementos antes mencionados, cuyas combinaciones forman figuras o formas específicas. 
Sin embargo, las formas encontradas en la naturaleza, como montañas, franjas costeras, sistemas hidrográficos, nubes, hojas, árboles, vegetales, copos de nieve y un sinnúmero de otros objetos no son fácilmente descritos por la geometría tradicional.

La geometría fractal provee una descripción y una forma de modelo matemático para las aparentemente complicadas formas de la naturaleza. Éstas poseen a veces una remarcable invariancia a la simplificación bajo los cambios de la magnificación, propiedad que caracteriza a los fractales como veremos más adelante.

Desde hace más de cien años, los matemáticos que se habían topado con los fractales los consideraban monstruos de una irregularidad insospechable. Los fractales fueron concebidos aproximadamente en 1890 por el francés Henri Poincaré; le continuó el sueco Helge von Kock con su "supercopo de nieve" en 1906, pero sus ideas fueron extendidas más tarde por dos matemáticos, también franceses, Gaston Julia y Pierre Fatou, hacia 1918. Se trabajó en este campo durante varios años, pero el estudio quedó congelado en los años veinte.

El estudio fue renovado a partir de 1974 en IBM y fue fuertemente impulsado por el desarrollo de la computadora digital. Pero no es hasta1977-1982 que el profesor francoamericano Benoit Mandelbrot, antiguo estudiante de 1' École Polytechnique y ex miembro del Club Bourbaki de París, explicó que precisamente esta irregularidad insospechable es lo que conforma a la naturaleza, y que por lo tanto es nuestra esencia misma: lo que nos constituye. En honor a él, uno de los conjuntos que investigó, y también uno de los más bellos, es conocido por su nombre. Otros matemáticos, como Douady, Hubbard y Sullivan trabajaron también en esta área, explorando más las matemáticas que sus aplicaciones. 


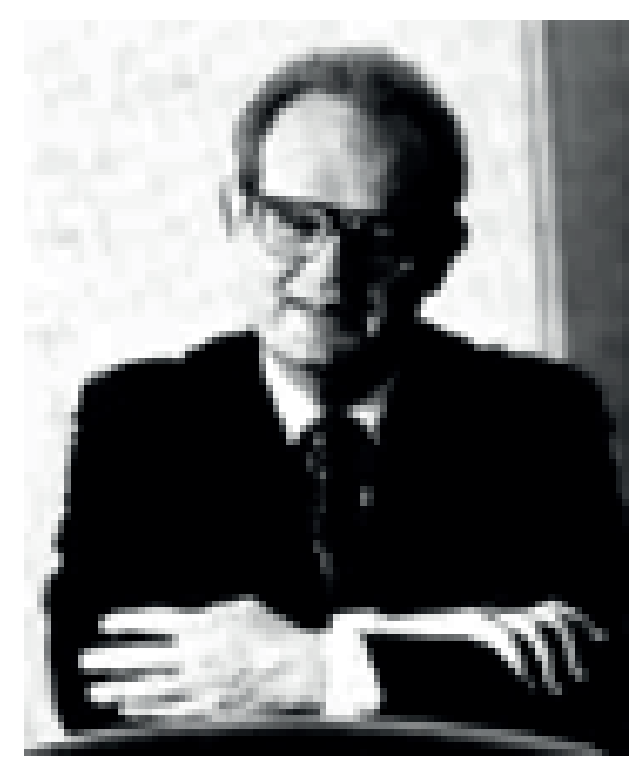

Benoit Mandelbrot

Desde la década de los setenta este campo ha estado en la vanguardia de los matemáticos contemporáneos. Investigadores como el Dr. Robert L. Devaney, de la Universidad de Boston, han estado explorando esta rama de la matemática con la ayuda de las computadoras modernas.

Para comprender el origen de la teoría que originó el descubrimiento de los fractales hemos de remontarnos al año 450 a. C. El filósofo Zenón de Elea propuso cuatro paradojas que parecían demostrar que el movimiento, tal como lo percibimos, es imposible. La más conocida por el público en general es la de "Aquiles y la tortuga". El enunciado, en pocas palabras, es:

Supongamos que Aquiles (el más veloz de todos los héroes griegos que sitiaron Troya) puede correr diez veces más deprisa que una tortuga, y supongamos que ambos toman parte en una carrera en la que la tortuga tiene una ventaja inicial de diez metros.

Zenón afirma que es imposible que Aquiles adelante a la tortuga, porque cuando Aquiles haya recorrido los diez metros que le separan de la posición de partida de la 
tortuga, ésta ya habrá avanzado un metro. Cuando Aquiles recorre este metro, la tortuga ha avanzado una décima de metro. Cuando Aquiles haya recorrido esta distancia, la tortuga ha avanzado una centésima de metro, y así hasta el infinito. Aquiles cada vez se aproxima más a la tortuga, pero nunca puede alcanzarla del todo.

El razonamiento es impecable, pero todos sabemos que, en realidad, Aquiles no tardará mucho en alcanzar a la tortuga. De hecho, dice la leyenda que Zenón murió atropellado por un carro, quizá pensando en que, con su ventaja, éste nunca le alcanzaría, aunque realmente fue juzgado y condenado a torturas hasta la muerte por conspirar contra el tirano local.

A esto se le llama paradoja: según el razonamiento lógico Aquiles no puede adelantar a la tortuga, pero la observación de la realidad nos dice que puede hacerlo y lo hace. Esta paradoja dejó perplejos a los matemáticos durante dos mil años, en parte debido a que parecía darse por supuesto que, dada una serie de números infinita (Ej. $10+1+1 / 10+1 / 100 \ldots)$ su suma tiene que ser infinita, y el tiempo empleado en recorrer la distancia representada por estos números también tiene que ser infinito.

La demostración de que esta suposición, tan obvia en apariencia, no era cierta se le atribuye al matemático escocés James Gregory hacia 1670. Es una demostración extremadamente sencilla. En la serie $10+1+1 / 10+1 / 100 \ldots$, si sumamos $10+1$ tenemos 11; si a esto le sumamos 1/10 tenemos 11.1; si a esto le sumamos 1/100 tenemos 11.11. Si añadimos un número infinito de términos, tendremos $11.11111 \ldots$, número perfectamente definido con una suma de números racionales: 11 + 1/9.

Ya tenemos representada la ventaja de los números infinitamente decrecientes de la tortuga sobre Aquiles: $11+1 / 9$. Por tanto, Aquiles adelantará a la tortuga en el 
tiempo que tarde en recorrer los $11+1 / 9$ metros. A las series infinitas cuya suma es finita, se les llama series convergentes.

Hagamos ahora una visualización de este fenómeno matemático. Imaginémonos una serie de cuadrados: el primero de $1 \mathrm{~cm}$ de lado, el segundo de $1 / 2 \mathrm{~cm}$, el tercero de $1 / 4 \mathrm{~cm}$, el cuarto de $1 / 8 \mathrm{~cm}$, etc. Imaginémonos que los colocamos uno pegado al lado del otro, en orden de mayor a menor. De este modo, tenemos una línea de infinitos cuadrados cada vez más pequeños uno al lado del otro. Todos ellos juntos ocuparían en total una longitud de $2 \mathrm{~cm}$ : el primero ocuparía la mitad de la longitud total $(1 \mathrm{~cm})$, el siguiente la mitad del resto $(1 / 2 \mathrm{~cm})$, el siguiente la mitad de lo que quede $(1 / 4 \mathrm{~cm})$, y así hasta el infinito.

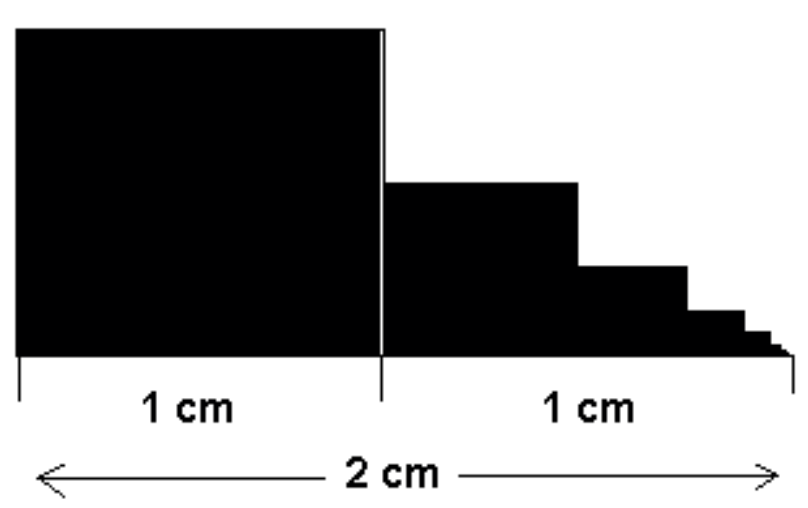

Los cuadrados se hacen extremadamente pequeños con mucha rapidez. El cuadrado número 27 tendría el tamaño aproximado de un átomo y, una vez que ocupa su puesto en la fila, todo lo que queda de los dos centímetros de la longitud total es un espacio de una anchura aproximadamente igual a la de un átomo. Pero en este insignificante espacio se amontonan un número infinito de cuadrados que siguen disminuyendo rápidamente de tamaño.

Éste cuadrado tiene un lado de aproximadamente $1 / 100.000 .000 \mathrm{~cm}$ de lado (2$27 \mathrm{~cm})$. Imaginemos que ampliamos cien millones de veces éste cuadrado y todos los 
demás. Ahora, el cuadrado vigesimoséptimo parece tener un centímetro de lado, el siguiente $1 / 2 \mathrm{~cm}$, el siguiente $1 / 4 \mathrm{~cm}$, etc. De esta ampliación resultaría una serie exactamente igual, tanto en tamaño como en número de cuadrados, a la serie original. En este caso, el cuadrado número 15 sería tan pequeño como el tamaño de un protón, pero podríamos ampliarlo para que pareciera tener $1 \mathrm{~cm}$ de lado, ...y tendría una cola de cuadrados todavía más pequeños, exactamente iguales tanto en tamaño como en número, a la serie original de partida. Podríamos seguir así eternamente y nunca acabaríamos. Contaríamos millones, trillones, quintillones de cuadrados, que siempre seguiríamos teniendo una cola de cuadrados exactamente igual a la serie original, toda ella situada en una extensión de dos centímetros.

Nos hemos centrado en la interpretación matemática de las series convergentes. Visualicémoslos desde el punto de vista de la geometría, y para ello usaremos el "supercopo de nieve" de Helge von Kock.

Tomamos un triángulo equilátero, dividimos cada lado en tres partes y construimos otro triángulo equilátero más pequeño en el tercio medio de cada lado, consiguiendo así una estrella de seis puntas. Dividimos ahora cada uno de los lados de los seis triángulos de la estrella en tres partes iguales y dibujamos ahora otro triángulo equilátero más pequeño en el tercio medio de cada lado. Seguimos dividiendo los lados y añadiendo triángulos eternamente...

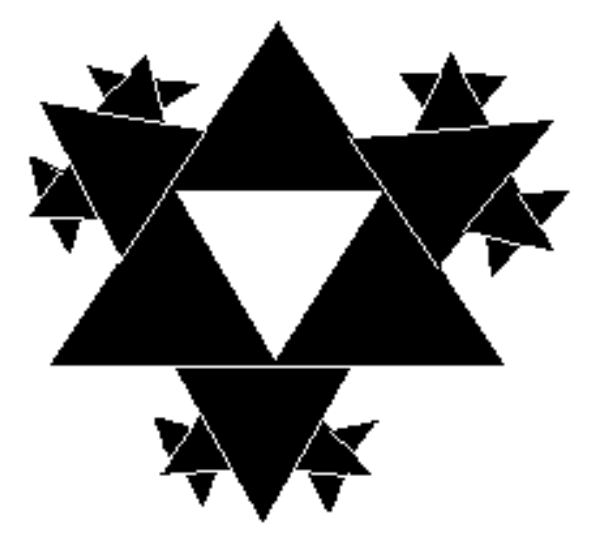

Supercopo de nieve de Kock

En la figura únicamente están desarrollados tres triángulos 
El perímetro de este supercopo de nieve forma una serie divergente, es decir, tiende al infinito. Sin embargo, las áreas de los triángulos que forman el supercopo forman una serie convergente y, por tanto, su suma es un número finito (concretamente 1.6 veces mayor que el área del triángulo de partida).

Por más que ampliemos la imagen en cualquiera de los triángulos de partida obtendremos triángulos igualmente complejos que los originales. A esta situación se le llama autosemejanza, y las figuras que cumplen esta condición ni siquiera cumplen las propiedades fundamentales de las figuras geométricas tradicionales. A este tipo de figuras se les denomina fractales.

¿Cuál es el origen de la palabra? Benoit Mandelbrot acuñó la palabra fractal derivándola del adjetivo latino fractus. El correspondiente verbo latino, frangere, significa romper, crear fragmentos irregulares.

La geometría fractal se dedica al estudio de las figuras con infinito detalle que describen algunos fenómenos naturales. Es la ciencia inherente de lo impredecible. Los fractales cumplen en general con tres características importantes:

1. Se basan en una geometría compleja y de infinito detalle; es decir, podemos aumentar miles de veces el lente de nuestra cámara, y seguiremos viendo tanto detalle, aproximadamente, como el que tenía la figura original.

2. La información que los genera es relativamente poca, y en particular se basan en un principio de retroalimentación de los resultados, llamados iteraciones, que dependen de los parámetros de entrada. Normalmente se manejan números complejos. (Un número complejo es aquel que se compone de una parte real y de una parte imaginaria, en otras palabras, se trata simplemente de un par de números con los que se trabaja siempre juntos, y para los que se definen una serie de reglas de 
juego. El ejemplo más típico de números tomados a pares lo constituye la descripción en un plano de sus puntos, o la posición en la superficie esférica de la Tierra, también dada con pares de valores: latitud y longitud).

3. La mayoría de los fractales cumplen con el principio de "similitud a sí mismos" (self-similar). Matemáticamente, es una figura geométrica compleja y detallada en estructura a cualquier nivel de magnificación. A menudo los fractales son semejantes a sí mismos, esto es, poseen la propiedad de que cada pequeña porción del fractal puede ser visualizada como una réplica a escala reducida del todo.

Un ejemplo de la naturaleza es un brécol. Al mirar el brécol, se observa que es una verdura con un tronco y con un grupo de "florecillas" en la parte superior. Pero curiosamente, al mirar con más cuidado, se observan tallos más delgados con sus respectivas "florecillas" que a su vez se constituyen por tallos aún más delgados y florecillas más pequeñas. Precisamente la característica de realizar "zooms" en los fractales se le conoce con el nombre de similitud de sí mismos. De la misma manera podemos observar un helecho compuesto de un tallo con sus respectivas hojas, una nube conformada de nubes más pequeñas, o bien una montaña constituida por montañitas.

Existen varios fractales que son ya famosos por sus características. El más famoso de todos ellos es el conjunto de Mandelbrot. El conjunto de Mandelbrot, como todos los demás fractales, es generado por iteraciones. Iteración significa repetir un proceso varias veces. En matemática, este proceso es, casi siempre, la aplicación de una función. Para el conjunto de Mandelbrot, la función involucrada es la función no lineal más simple de imaginar, $\mathrm{z} 2+\mathrm{c}$, donde c es una constante. Veremos más tarde el valor exacto de c.

La fórmula que describe al conjunto de Mandelbrot es: 
Znuevo $=$ Zanterior $x$ Zanterior $+\mathrm{c}$

Donde z es un número complejo (Ej. 9+3i, o lo que es lo mismo, el par de números 9 y 3) y c es una constante (Ej. -0.122 + 0.745i, es decir, el par de números -0,122 y 0,745). En realidad, y como apunte para los interesados, al conjunto de Mandelbrot se le considera una especie de diccionario que describe a otros fractales, llamados los conjuntos de Julia.

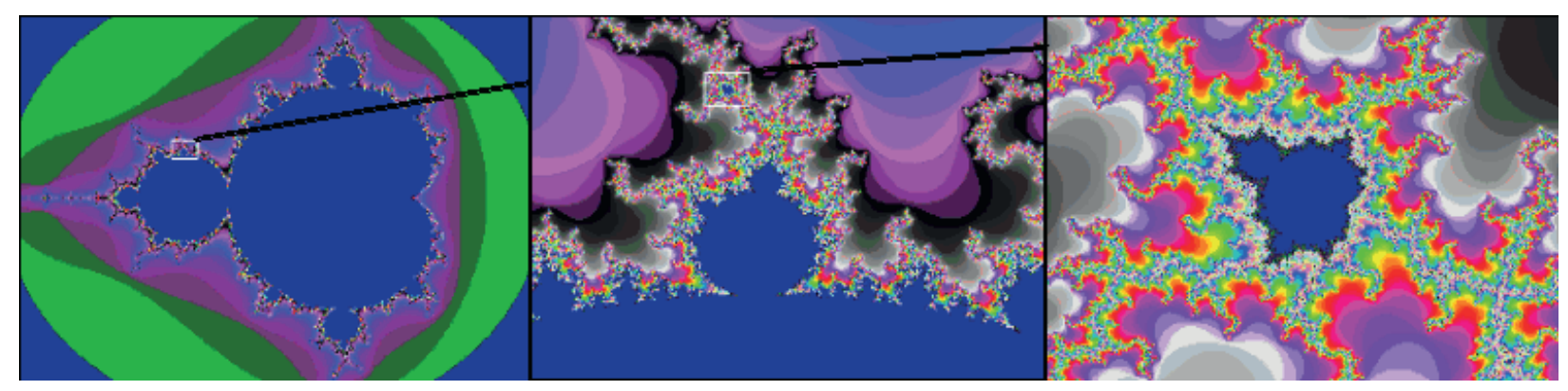

A la izquierda, el conjunto de Mandelbrot. Al centro, una ampliación de la muestra tomada a la imagen de la izquierda. A la derecha, una ampliación de la muestra tomada de la imagen del centro. Vemos que aparece de nuevo una imagen igual a la primera. A esta propiedad se le llama, "self-similar".

Una de las principales preguntas en esta área de las matemáticas es: ¿Cuál es el destino de las órbitas típicas? (se llama órbita, en este caso, al propio desarrollo de la función al efectuar iteraciones) ¿Convergen o divergen? ¿Son cíclicas, constantes o se comportan erráticamente? En realidad, el conjunto de Mandelbrot es una versión geométrica de las respuestas a estas preguntas.

Comencemos con unos ejemplos. Supongamos que c=1. Luego, si elegimos la semilla, o valor inicial de $\mathrm{Z} 0=0$, la órbita es: 
$\mathrm{Z} 0=0$

$\mathrm{Z1}=1=\mathrm{Z} 2+1$

$\mathrm{Z} 2=2=12+1$

$\mathrm{Z} 3=5=22+1$

$\mathrm{Z} 4=26$

Z5 = NÚMERO GRANDE

Z6 = NÚMERO MÁS GRANDE

Y notamos que esta órbita tiende al infinito. Sería una órbita divergente.

Ahora supongamos que $c=0$. La órbita de la semilla 0 es muy diferente: esta órbita permanece constante para todas las iteraciones.

$\mathrm{Z} 0=0$

$\mathrm{Z} 1=0$

$\mathrm{Z} 2=0$

Que sería una órbita constante con su valor en cero.

Si ahora suponemos que c=-1. Para la semilla 0, la órbita es:

$$
\begin{aligned}
& Z 0=0 \\
& Z 1=-1 \\
& Z 2=0 \\
& Z 3=-1 \\
& Z 4=0 \\
& Z 5=-1
\end{aligned}
$$

Ahora vemos que la órbita va de 0 a -1 y viceversa, con un ciclo de periodo 2 . También tendríamos una órbita cíclica, en este caso de dos valores. 
Matemática - Fractal - Geometría

Para entender el destino de las órbitas, es más fácil proceder geométricamente. A menudo, se consigue más información del destino de las órbitas con un gráfico que representa los resultados de cada iteración para un cierto valor del parámetro c. En los gráficos siguientes mostramos los resultados de las órbitas de $Z 0=0$ para $c=-1.1$, $\mathrm{c}=-1.3, \mathrm{c}=-1.38, \mathrm{c}=-1.9$

Para $\mathrm{c}=-1.1$ vemos que la órbita se aproxima a un ciclo de periodo 2

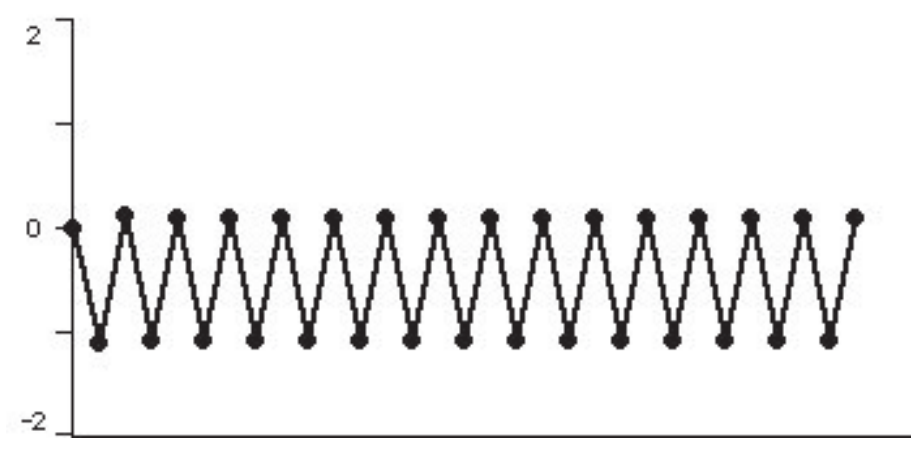

Para $\mathrm{c}=-1.3$ la órbita tiende a un ciclo de periodo 4

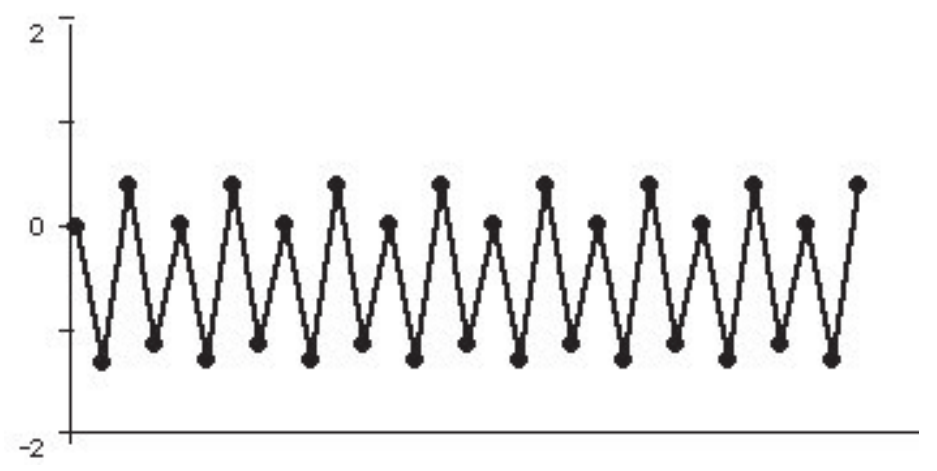

Para c $=-1.38$ vemos un ciclo de periodo 8

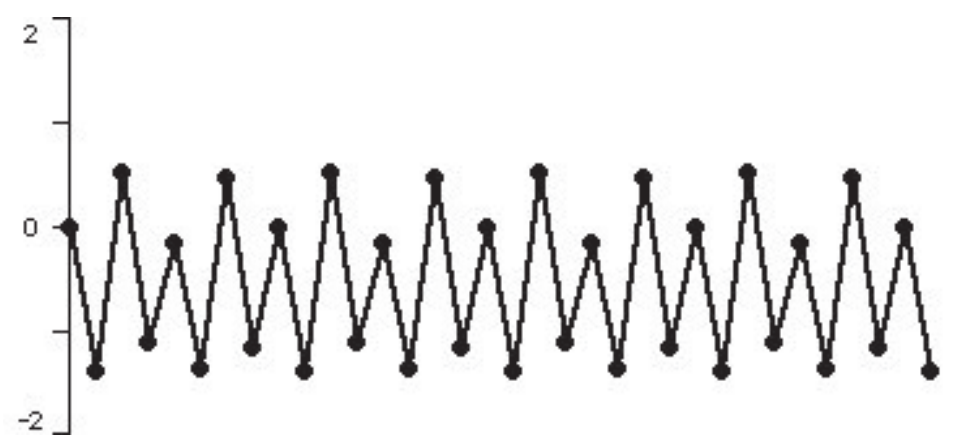

23

ISSN: 1575-2844 · DOI: http://dx.doi.org/10.15178/va.1999.11.12-32 
Cuando c=-1.9 no hay ningún ciclo aparente para la órbita

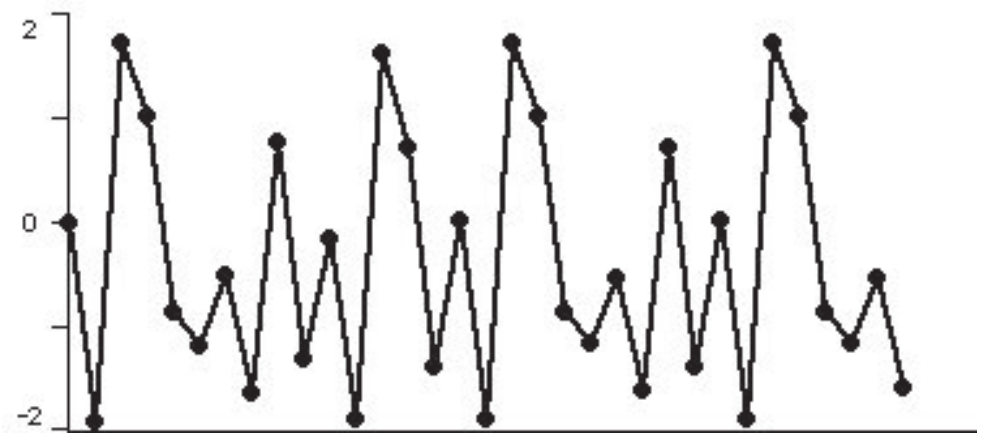

Los matemáticos utilizan la palabra caos para este último fenómeno.

Antes de proceder a calcular el conjunto de Mandelbrot, hagamos una observación obvia. Bajo la iteración de $\mathrm{z} 2+\mathrm{c}$, la órbita 0 (es decir $\mathrm{Z} 0=0$ ) tiende al infinito o no $(\mathrm{Ej}$. con $c=1$ tiende al infinito, es divergente). Cuando la órbita no va al infinito, ésta se comporta de varias maneras. Puede ser constante (Ej. c=0), cíclico (Ej. c=-1) o caótico (Ej. c=1.9), pero la observación fundamental es que ésta órbita consiste en una dicotomía: a veces la órbita va hacia el infinito, y otras veces, no. El conjunto de Mandelbrot es el gráfico que representa esta dicotomía, es decir, los números que al iterarse tienden a infinito o no cuando la semilla es $\mathrm{Z} 0=0$. Por lo tanto, el conjunto de Mandelbrot es un registro del destino de la órbita de $\mathrm{Z0}=0$ bajo la iteración $\mathrm{z} 2+\mathrm{c}$. La observación de que la órbita $\mathrm{Z} 0=0$ bajo $\mathrm{z} 2+\mathrm{c}$ tienda al infinito o no es fundamental para entender el gráfico.

Entonces, ¿cómo es posible que el conjunto de Mandelbrot sea un gráfico en dos dimensiones, o plano, en vez de lineal? La respuesta es que, en vez de considerar sólo los valores reales de c, también consideramos los números complejos, representando el conjunto de Mandelbrot en un gráfico cuyo eje horizontal representa a los números reales y el vertical a los imaginarios.

Por ejemplo, la órbita de $\mathrm{Z} 0=0$ bajo c=i está dada por: 
$\mathrm{Z} 0=0$, es decir, el par 0,0

$\mathrm{Z} 1=\mathrm{i}$, es decir, el par 0,1

$Z 2=-1+i$, es decir, el par $-1,1$

$\mathrm{Z3}=-\mathrm{i}$, es decir, el par $0,-1$

$Z 4=-1+i$, es decir, el par $-1,1$

$\mathrm{Z} 5=-\mathrm{i}$, es decir, el par $-1,0$

$Z 6=-1+i$, es decir, el par $-1,1$

Y vemos que la órbita eventualmente se convierte en un ciclo de periodo 2.

Si cambiamos c por 2i, entonces la órbita se comporta de otra manera:

$\mathrm{Z} 0=0$

$\mathrm{Z} 1=2 \mathrm{i}$

$Z 2=-4+2 i$

$\mathrm{Z3}=12-14 \mathrm{i}$

$\mathrm{Z} 4=52-334 \mathrm{i}$

$\mathrm{Z} 5=$ GRANDE (LEJOS DEL ORIGEN)

Z6 $=$ MÁS GRANDE

Y vemos que esta órbita tiende al infinito en el plano complejo (los números de la órbita se alejan cada vez más del origen en una serie divergente).

El conjunto de Mandelbrot introduce algo de geometría en la observación fundamental mencionada anteriormente. La definición precisa es: El conjunto de Mandelbrot M, consiste de todos aquellos valores (complejos, o pares de valores que representan puntos de un plano) de $\mathrm{c}$ cuyas órbitas de $\mathrm{Z} 0=0$ bajo $\mathrm{z} 2+\mathrm{c}$ correspondientes no escapan al infinito. De nuestros cálculos anteriores, vemos que 
$c=0,-1,-1.1,-1.3,-1.38$ e i pertenecen al conjunto Mandelbrot, mientras que $c=1$ y c=2i no pertenecen.

Para su representación gráfica siempre se hace $Z 0=0$. La pregunta fundamental a contestar es si después de un cierto número de iteraciones, dada la constante c, el resultado tenderá a infinito o no.

Si el resultado es infinito, entonces la parte del plano que corresponde a los números complejos c se colorea de un color (normalmente negro).

Si el resultado tiende a un valor finito, la parte del plano que se corresponde con esos pares de valores que representa c se colorea de otro.

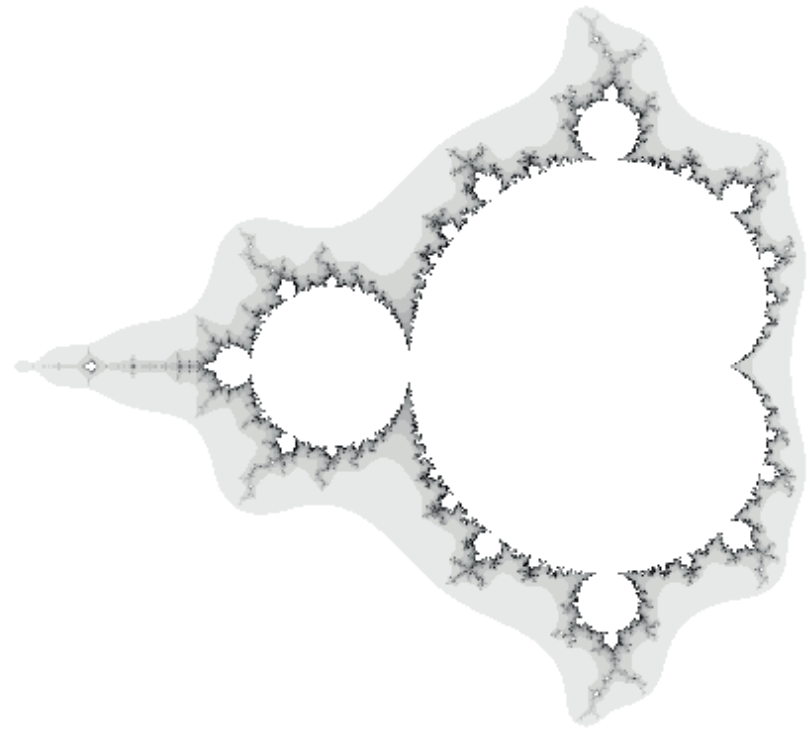

Conjunto de Mandelbrot en escala de grises.

Sólo están en negro los valores cercanos al límite.

También se puede escoger un color según el número de iteraciones necesarias antes de llegar a un resultado no infinito. Al final, el plano complejo queda como la siguiente figura: 


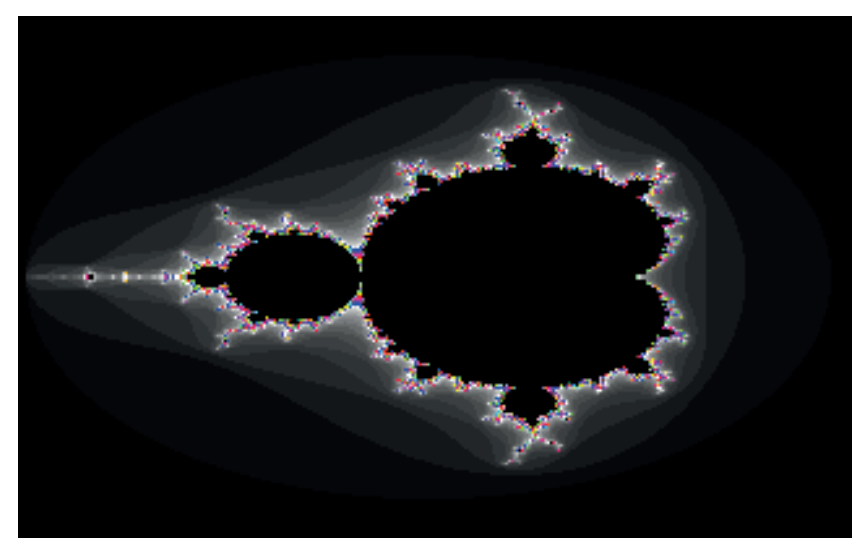

Conjunto de Mandelbrot a color.

Los colores se escogen en función del número de iteraciones realizadas

Otro fractal muy famoso es el triángulo de Sierpinsky. Este fractal se construye al utilizar un triángulo como parámetro inicial, y posteriormente iterando las siguientes reglas:

1) Se escala el objeto a la mitad

2) Se repite el objeto tres veces: una en la parte inferior izquierda, otra en la parte inferior derecha y la última en la parte central superior.

Triángulo de Sierpinsky obtenido a partir de un cuadrado
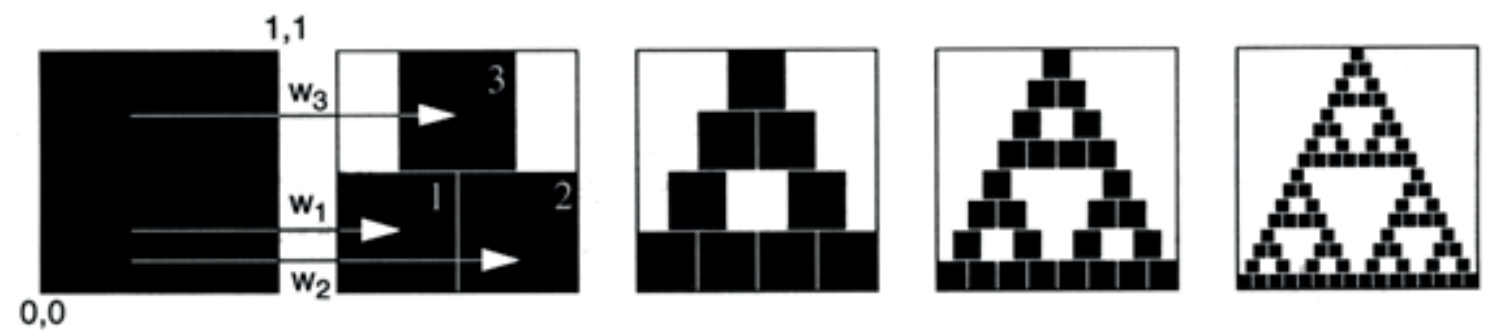

Después de una primera iteración se obtiene como resultado una figura construida por tres triángulos. Después de la segunda iteración serán nueve. Después de la tercera serán 27 , etc. 


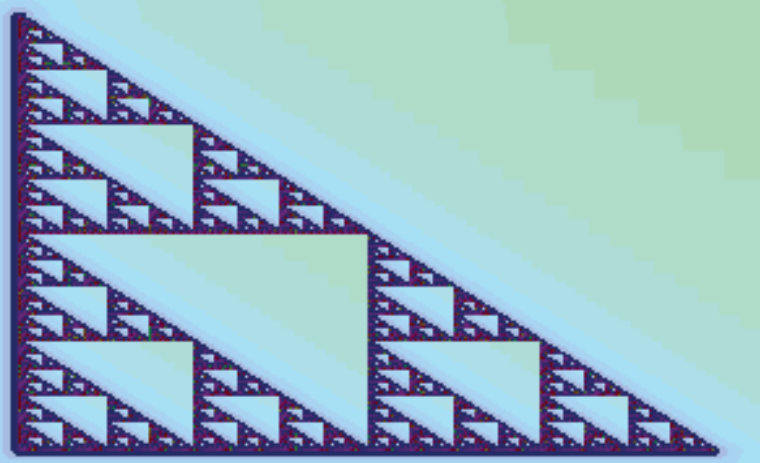

Triángulo de Sierpinsky obtenido a partir de un triángulo rectángulo

Esta figura además tiene varias características dignas de estudio. No importa con qué figura se comienza (un triángulo, un cuadrado, una cara, un barco, etc) Si se siguen las reglas, después de un cierto número de iteraciones siempre se obtendrá la misma figura: El triángulo de Sierpinsky.

Es un objeto con dimensión fractal de 1.58. Esto quiere decir que es un objeto que tiene dimensión mayor a una línea recta (dimensión=1) y una dimensión menor a la de un cuadrado (dimensión=2). Desarrollemos algo más este concepto.

Cuando aprendemos las primeras nociones de geometría nos enteramos de que el punto no tiene dimensiones, que la línea es unidimensional, el plano bidimensional y los sólidos tridimensionales. Podemos completar más aún si consideramos que si un sólido tiene cierta duración y existe en el tiempo es tetradimensional. Pero todas estas dimensiones son números enteros: $0,1,2 \ldots$

Sin embargo, Mandelbrot demostró que el límite, por ejemplo, del supercopo de nieve de Kock es tan borroso y presenta unos cambios de dirección tan bruscos en cada punto, que no podía ser considerado una línea en el sentido normal, sino algo 
que no es exactamente una línea ni tampoco un plano. Su dimensión ocupa un lugar intermedio entre 1 y 2, concretamente $\log 4 / \log 3$, aproximadamente 1.26.

La característica que fue decisiva para llamarlos fractales fue su dimensión fraccionaria. No tienen dimensión uno, dos o tres como la mayoría de los objetos a los cuales estamos acostumbrados. Los fractales tienen usualmente una dimensión que no es entera, ni uno ni dos, pero muchas veces entre ellos, por ejemplo: 1.79.

Por lo tanto, ya que existen fractales con dimensión 4.23, 5.02, etc., ¿entonces en la naturaleza existen objetos con dimensión fraccionaria? ¿Están entre una línea y el plano o bien entre el plano de dos ejes y el plano de tres ejes (entre un cuadrado y un cubo)?
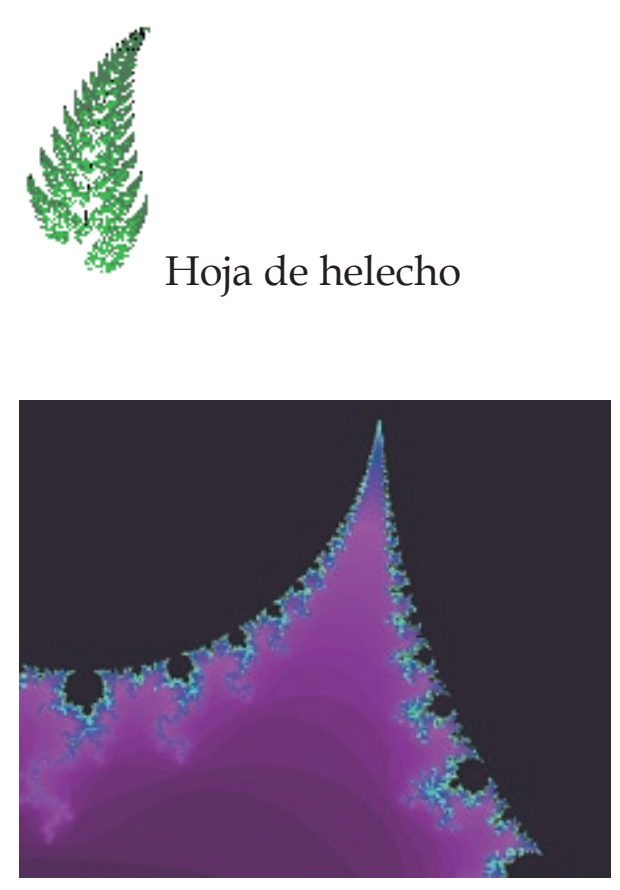

Detalle del conjunto Mandelbrot

Es importante reconocer que los fractales verdaderos son una idealización: ninguna curva en el mundo real es un fractal verdadero. Sin embargo, tampoco son ejemplos monstruosos de formas geométricas fruto de la imaginación de los matemáticos. En realidad, se parecen más a los objetos del mundo real que las curvas y planos simples 
y uniformes de la geometría idealizada. Éstos sí que son productos de la imaginación, aunque no hay que olvidar que los objetos reales son producidos por procesos que actúan sólo sobre un rango de escalas finitas. En otras palabras, los objetos reales no tienen la infinita cantidad de detalles que los fractales ofrecen con un cierto grado de magnificación.

Uno de los muchos pensamientos extraños que sugiere el conjunto Mandelbrot es que, en principio, podía haberse descubierto cuando la especie humana aprendió a contar. En la práctica, puesto que una imagen de pocos aumentos puede implicar miles de millones de cálculos, en modo alguno podría haber sido ni entrevista antes de que se inventaran los ordenadores. Para hacer las imágenes de este texto hubiera sido necesario que toda la actual población del mundo hubiera estado haciendo números día y noche durante años, sin cometer ni un error, para multiplicar billones de números de cien cifras.

No está muy claro quién fue el genio que tuvo la idea de poner colores a la velocidad de crecimiento, o número de iteraciones mínimo para considerar que tiende a infinito, de las series que resultan ser divergentes (quizá el propio Mandelbrot), pero las convierte en auténticas y fantásticas obras de arte. ¿Quién se iba a imaginar que una ecuación tan absurdamente sencilla podría generar esta, literalmente, infinita complejidad y esta sublime belleza? 


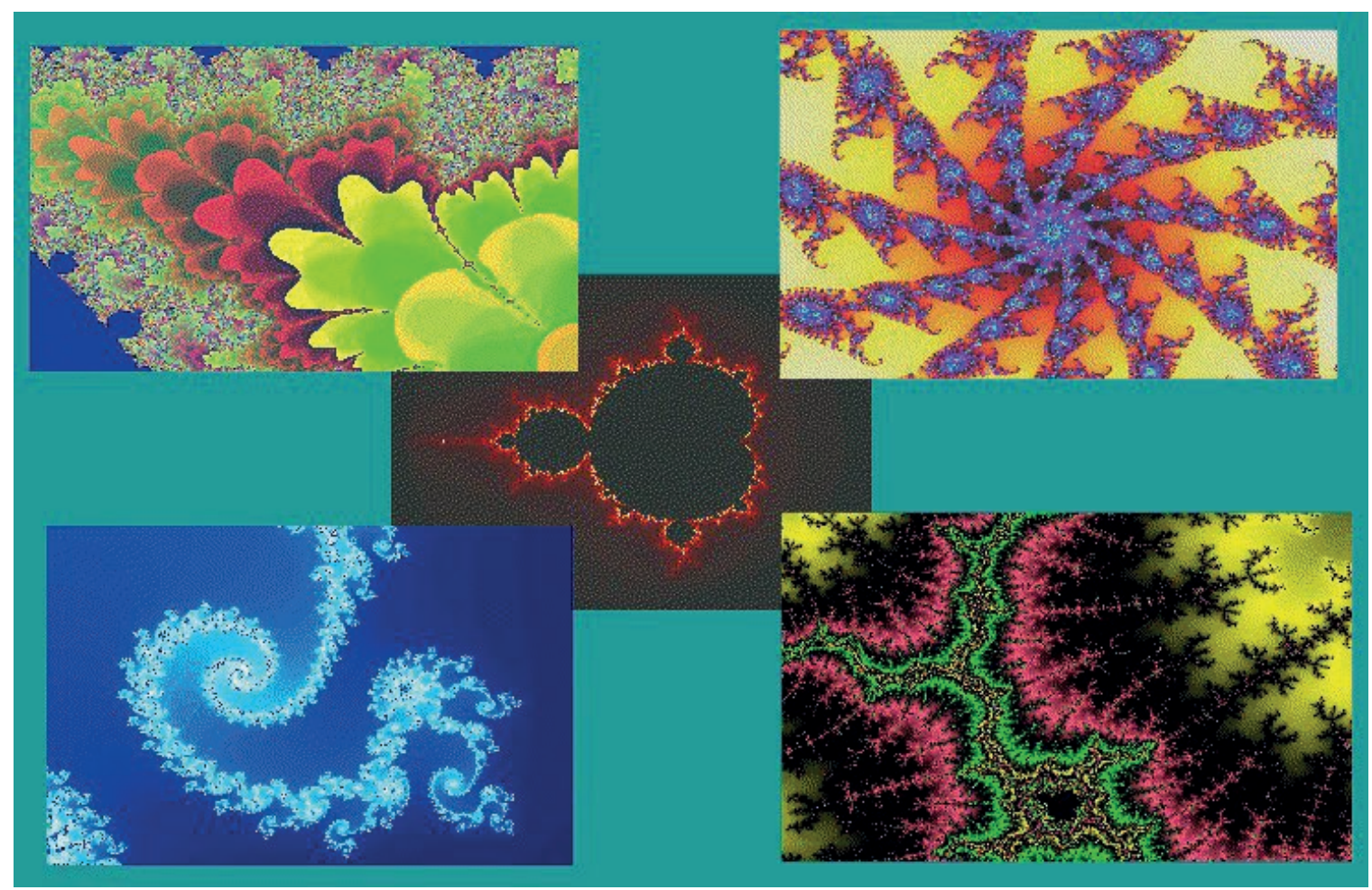

Unos ejemplos que ilustran la belleza intrínseca de la Matemática Fractal

Existe aún mucho material por ahondar en la geometría fractal, que además está íntimamente relacionada con la teoría del caos. El caos es un comportamiento que, aparentemente, es impredecible y que surge dentro de un sistema determinista cuando éste se hace muy sensible a las condiciones iniciales. Por ejemplo, la turbulencia en una cañería de agua, el desorden en las señales enviadas a los nervios del corazón o la histeria celular conocida con el nombre de cáncer son ejemplos de aleatoriedad. Lo importante es que ya se han comenzado a aplicar los conocimientos teóricos, como, por ejemplo, para la comprensión fractal de imágenes y la esteganografía, o técnica de ocultar o encriptar información entre otros datos que hacen de "envoltura". Para la comunidad de la computación gráfica, ahora ya se puede pensar que los fractales no solamente forman texturas espectaculares de mármoles, nubes, triángulos extraños, caracolas, pétalos de flores o copos de nieve con detalle infinito. También son una poderosa herramienta para el estudio de las formas de la naturaleza, la criptografía y esteganografía, o los nuevos campos de las 
matemáticas, además de abrir un prácticamente infinito abanico de posibilidades para el descubrimiento de nuevos estudios para la ciencia.

No olvidemos que también el láser fue limitado en sus aplicaciones desde su descubrimiento hasta hace bien poco, y hoy en día se ha convertido en una herramienta indispensable para todos en la mayoría de las aplicaciones tecnológicas, desde el CD-ROM hasta los punteros, por no mencionar su utilidad en medicina, tecnología o comunicaciones. Y sin embargo, el gran público desconoce, no ya su funcionamiento, sino lo que es realmente, al igual que los fractales.

Fuentes

http://www.quanta.net/zfractal.htm

"El espectro del Titanic" de Arthur C. Clarke. Plaza \& Janés Editores, 1991

"El secreto del universo y otros ensayos" de Isaac Asimov. Ediciones Temas de Hoy, 1991

"La última partida" de Tim Powers. Ediciones Martínez Roca, 1993 puntos de cuerpo. Entre párrafos se dejará una línea de cortesía y antes y después de cada epígrafe y subepígrafe. 